\title{
Using VineUp to Match Students with Alumni Industry Mentors in Engineering: A Pilot Study
}

\author{
Colleen Halupa ${ }^{1,2}$ \& Matthew Henry ${ }^{1}$ \\ ${ }^{1}$ Center for Innovation in Teaching and Learning, LeTourneau University, Longview, TX, USA \\ ${ }^{2}$ College of Graduate Health Studies, A.T. Still University, Kirksville, MO and Mesa, AZ. USA \\ Correspondence: Colleen Halupa, Center for Innovation in Teaching and Learning, LeTourneau University, \\ Longview, TX, USA \& College of Graduate Health Studies, A.T. Still University, Kirksville, MO and Mesa, AZ. \\ USA. Tel: 1-903-233-4071. E-mail: colleenhalupa@ letu.edu or chalupa@atsu.edu
}

Received: August 30, 2015

doi:10.5430/ijhe.v4n4p105

\begin{abstract}
Accepted: September 11, 2015
Online Published: September 17, 2015
\end{abstract}

URL: http://dx.doi.org/10.5430/ijhe.v4n4p105

\begin{abstract}
This pilot study evaluated users' perceptions of the effectiveness of the VineUp platform to match mentors from a large United States manufacturing firm and mechanical engineering students in an honors program at a small private university. Four mentor/mentee pairs were surveyed and interviewed at the end of the nine-month program. Although the VineUp platform was effective in matching mentor/mentee pairs, participants felt the process could have been done with an alternate method that was more cost effective.
\end{abstract}

Keywords: Mentoring, E-mentoring, Student-industry partnerships, Value in mentoring

\section{Introduction}

The purpose of this qualitative pilot study was to qualitatively evaluate the satisfaction of undergraduate engineering students and industry mentors from industry as part of a small private university's honor college program. VineUp is a professional social media program that matches mentors and mentees using personal and professional/work habits and characteristics. In this study VineUp was exclusively used to match mentors and mentees with "like" characteristics to foster an effective mentoring relationship. After matching occurred, mentors and mentees were free to use any tools at their disposal to foster the relationship (telephone, Skype, email, etc.). Nine months after the mentoring relationship was established, both the mentors and the students participated in a 15-minute targeted interview to determine their satisfaction with the mentoring program and to identify whether or not the match-up provided by Vine-Up was effective. This pilot study was funded by a grant by the company that employed the mentors. The company also gave mentors time to conduct mentoring sessions.

\subsection{Background}

Pinelli, Hall \& Brush (2013) note, "In the $21^{\text {st }}$ century innovation and engineering may hold the key to economic growth, prosperity, security and competitiveness of the Unites States" (p. 5). According to the National Academy of Engineering, the gap between industry and engineering education is continually widening. Researchers (Kapitzke \& Hay, 2012; Kim et al., 2014) have noted the lack of true skills for employment is a global issue.

\subsection{Research Purpose and Goals}

The overarching purpose of this qualitative pilot study was to evaluate the satisfaction and perceptions on industry partners and mechanical engineering students at an honors college towards an e-mentoring program. A second purpose was to evaluate the perceived effectiveness of the VineUp platform in facilitating an effective mentor-mentee match.

\section{Literature Review}

\subsection{Mentoring}

Mentoring is a method to foster individual growth. There can be many types of mentoring relationships including youth mentoring, faculty-to-student mentoring and workplace mentoring. Although mentoring has been being conducted since the beginning of time, the seminal study on mentoring was conducted by Levinson, Darrow, Levinson, Klein and McKee in 1978 who followed 40 men throughout their lives to examine the effect of mentoring relationships in human development. Levinson et al. described a mentor as a "guide, teacher, counselor and developer of skills who 
facilitate the realization of a Dream: (p. 98 as quoted in Lillian, Eby, Rhoes \& Allen, 2011). In general, mentoring was found to lead to success; however, some experiences may be life-altering and some may have little effect or even be negative (Levinson et al., 1978). Mentoring is a process; although the mentee is thought to have the greatest benefit, there may also be benefit for the mentor. The mentor-protégé relationship is similar to a role model-observer relationship and may be formally or informally initiated (Lillian et al., 2011).

\subsection{Effective Relationships and Satisfaction in Mentoring}

Straus, Johnson, Marquez and Feldman (2013) looked at the effectiveness of mentor/mentee relationships in 2013 in academic health centers. They conducted semi-structured interviews based on grounded theory with 54 faculty members. They identified several key characteristics of effective mentors and mentees:

Mentors:

- Must be altruistic

- Must be engaged and focused during mentoring sessions

- Must have professional experience

- Must be accessible and able to identify mentees' strengths and weaknesses

- Should provide emotional support

- Should advise mentees of potential pitfalls

- Should provide strategic advice

Mentees

- Must be open to feedback

- Must be active listeners

- Must be responsible

- Must value timelines

- Must drive the relationship (Straus et al., 2013, p. 84-55)

According to Straus et al. (2013), ineffective mentoring relationships suffer from "poor communication, lack of commitment, personality differences, perceived competition, conflicts of interest and the mentor's lack of experience (p. 87). They also note mentors and mentees must share "mutual goals, respect, trust and commitment" (p. 87) in order for the relationship to be satisfying and effective.

\subsection{Technology in Mentoring}

Mentoring that occurs using electronic forms of communication is called e-mentoring or virtual mentoring (Kasprisin, Single, Single \& Muller, 2003). This can include e-mail, instant messaging, chat, virtual meetings and social media. These tools have allowed mentoring relationships to flourish beyond geographical boundaries and allows access to those who may have not been able to participate in effective mentoring relationships previously. Kaspirin et al. in 2003 noted online mentoring is not always a cheaper option; however, today, many of these options such as Skype, email, social media and even programs such as GoToMeeting are free to users. Cost is incurred if a standardized or validated matching process between mentor and mentee is utilized. These incurred costs may be time, cost for instruments (such as Myers Briggs instrument) or the cost of databases to manage matching and communication in a single platform.

\section{Industry-Student Mentoring}

Beaty, Waters, Dziuvenis \& Feldman (2014) described the positive impacts of student/industry partnerships in the building design industry. They note these partnerships benefit employers by allowing them to understand the abilities of students who may apply for employment with their organization after graduation. Lam \& Adams (2014) found a mentoring program between managerial or executive-level professionals and undergraduate business students enhances student learning.

\subsection{Industry Mentoring Using Technology}

Khan \& Gogos (2013) discussed a mentoring program which partnered professionals from the biotechnology industry and students at the University of Maryland University College. In this program, masters' level students were paired with mentors at various companies. They started with 19 mentee pairs in 2009 and increased to 46 pairs by the fall of 2011. They found these mentoring relationships were effective in both student satisfaction, volition and motivation 
evidenced by the graduation rate of students who had been mentored compared to those that had not. The researchers noted such programs "bridge the gap between industry and academia" (p. 89). In this program evaluated by Khan and Goges, students were paired with mentors based on area of specialization, geographical location and shared interests; a standard or validated process for matching the mentee pairs was not used. Students were matched based on what they had in their applications.

Khan \& Gogos (2013) noted making the right match was challenging because pairing based on applications resulted in incompatible pairings due to the lack of motivation on the part of some of the mentees and because some mentees wanted general versus specific advice. They modified their matching procedures to include an interview before matching. Each mentee was required to prepare a professional action plan, while the mentors submitted their biographies. These were exchanged at the first meeting. An online platform was provided for interactions to occur and the groups met via email, chat, videoconferencing or telephone. There were occasional face-to face meetings with pairs in the same geographical location. The mentees were in various stages of their degree programs. Khan and Gogos found those students in the mentoring program completed more classes than those who were not; however, the differences in GPA were not significant. In addition, graduation rates in the mentee group were increased and they completed their degrees in less time than non-participants. Mentee satisfaction ratings overall were higher than those of the mentors.

Josh McCarthy at the University of Adelaide (UA) in Australia discussed an e-mentoring project between UA, Penn State (PS) and industry partners in digital media. The industry partners mentored the graduate UA students, while these UA students mentored PS undergraduate students. Mentoring was done using Facebook. This platform was used because it was something students were already using; private groups could be created and images and files could be uploaded and shared. It is also available in 70 languages to support international students. To decrease the potential for cyberbullying, which occurs frequently on social media platforms, specific administrative rules were used. Mentors and students were guided to create new profiles for this project so personal information was not shared. Overall $83 \%$ of the students were satisfied with the feedback they received from associated professionals (McCarthy, 2012).

\section{VineUp}

VineUp is a social media platform created in Great Britain specifically to match mentors and mentees using like characteristics, similar to the online dating platforms such as e-Harmony. Each VineUp participant completes a profile that outlines professional and personal likes and behaviors. A private online community can be created to facilitate mentoring. The site also lists career development opportunities and jobs. VineUp is integrated with Facebook and Linked-In and it can also be used to connect alumni with the university to facilitate the mentoring of students. The purchaser of the VineUp platform owns all of the data on members. Users of this platform include the University of St. Andrews in Scotland, the United Nations, the American Film Institute, Oxford International College, the University of Glasgow and many more (VineUp, 2015).

The matching criteria for mentees and mentors that was used for this research project include the following:

- Bringing a product to market

- Business Acumen

- Business Strategy

- Career planning

- Career Transition

- Coaching

- Concept development

- Conflict Management

- Corporate Politics

- Decision Making

- Developing Leads

- Down-Sizing

- E-Commerce

- Entrepreneurship 
- Executive Presence

- Expansion

- Fundraising

- Influencing Others

- Innovation and Creativity

- Interpersonal Communication

- Leadership

- Lean \& Agile

- Managing Change

- Managing Others

- Mergers and Acquisitions

- Negotiating

- Pivoting

- Planning and Organization

- Presentations

- Public Speaking

- Project Management

- Sales

- Self-Awareness

- Sleeping In

- Social Strategy

- Startups

- Targets and Goal Setting

- Technical Specialist

- Work / Life Balance

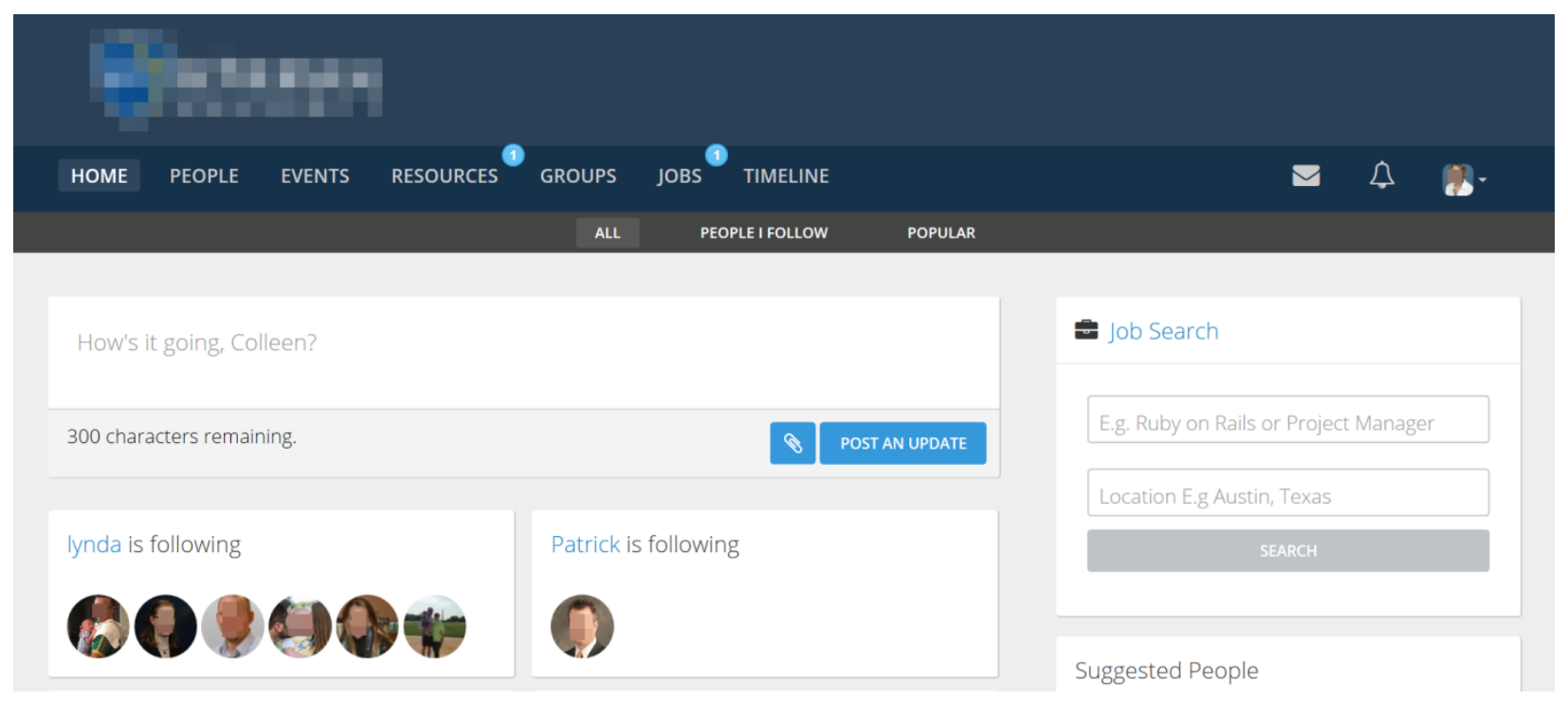

Figure 1.0 VineUp Home Page 


\section{Method}

The purpose of this research study was to assess industry mentor and mentee satisfaction with an e-mentoring program as well as to assess perceived effectiveness and satisfaction with VineUp, a social media mentoring program in an honors college. Volunteers from a large U.S. manufacturing firm were solicited and the company provided a grant for the VineUp software. All mentors were alumni of the university the mentees were attending. Student volunteers were recruited from an honors college at a small private university in Texas.

This was a pilot study and the initial group for this project consisted of five mentors and five students. Two mentors and one student opted not to participate after the initial study was started, so the final sample size for this pilot study was three mentors and four students. Members participated in an orientation meeting and both the mentors and mentees were given a mentoring guide that outlined program goals as well as general goals for mentors and mentees. The guide also discusses the stages of mentoring relationships, goal setting, mentoring skills, program expectations for mentees and included a list of sample questions mentees could ask their mentors to begin the relationship. The mentoring guide included worksheets for students to set professional and personal goals for the mentoring relationship. All participants were given a copy of an article on virtual mentoring by Zey (2011) which outlines the challenges and best practices for e-mentoring.

All participants created their profile on the VineUp software and completed the questions for proper matchup of professional and personal habits based on the 40 characteristics measured by VineUp. Mentors also uploaded biographies. The student mentees were responsible for reviewing the characteristics of the mentors and selecting one. The students contacted the mentors through VineUp and in order for the relationship to be initiated, the mentor had to accept the mentee through the VineUp program. Although VineUp is a professional social media platform, the participants were not required to pursue the mentoring relationship through VineUp. They were allowed to choose any method of contact that was agreed upon since the intent of this study was only to evaluate the matching process and participant perceptions of the VineUp software. The mentors and mentees met for nine months beginning in September 2014.

In June 2015, all mentors and mentees were contacted to participate in a short survey and a semi-structured interview. The survey instrument scale was strongly disagree, disagree, neutral, agree and strongly agree. Informed consent was obtained; all of the remaining students (4) and mentors (3) participated in the interviews. Means and descriptive statistics were calculated for the survey questions and the interview responses were evaluated for common and contrasting themes.

\section{Context and Setting}

All mentors and mentees in this pilot study were male. This was reflective of larger number of males than females in the engineering field. All mentors had degrees in mechanical engineering and were employed by a large U.S. manufacturing firm. All mentees were mechanical engineering students at a small private university. This university has a student population of approximately 2,500 students; approximately 50\% are residential students and the remainder are online students. The university offers undergraduate and graduate programs in engineering, psychology, education, business, humanities and aviation. There are approximately 850 students in the engineering programs including mechanical, civil and biomedical specialties.

\section{Results}

Both the mentees and mentors completed a brief Likert scale questionnaire to rate their perceptions of the relationship and the VineUp software. The means for relevant questions are listed in Table 1.0 and 2.0 below. Overall both groups were satisfied with the mentoring relationships, but overall, they were neutral to slightly positive in their perceptions of the VineUp software. The means for each group were evaluated. The largest difference in responses was to the question "I would recommend VineUp" where the mentee mean was 3.75 compared to the mentor mean of 3.0; this difference is not statistically significant because of the small sample size, but is notable. In addition, this group demonstrated more interest in the relationship and general career mentoring rather than goal setting. 
Table 1. Mentor and Mentee Perceptions of Mentoring Relationship

\begin{tabular}{ll}
\hline Question & Mean \\
\hline The mentoring relationship was effective. & 4.00 \\
My mentor (or mentee) had similar personal and ethical characteristics. & 4.42 \\
My mentor (or mentee) and I had similar professional habits/characteristics. & 4.30 \\
My mentor (or mentee) met with me as scheduled. & 4.50 \\
My mentor (or mentee) and I communicated effectively. & 4.16 \\
My mentor (or mentee) and I set specific goals & 3.50 \\
Specified goals for the mentoring relationship were met. * & 4.04 \\
I would select this mentor (or mentee) again for a mentoring relationship. & 4.15 \\
I did gain something from this mentoring relationship.* & 3.83
\end{tabular}

Note: * these two items were reverse coded

Table 2. Mentor and Mentee Perception of VineUp Software

\begin{tabular}{ll}
\hline Question & Mean \\
\hline The VineUp program was easy to use. & 3.75 \\
The VineUp program effectively matched me with a mentor/mentee. & 3.79 \\
I would recommend the VineUp software to others. & 3.37 \\
The match of mentor-mentee was better with the VineUp software than it would have been if we & 3.83 \\
had been matched up randomly. & \\
The match of mentor-mentee was better with the VineUp software than it would have been if we & 3.58 \\
had completed a small interest/habits survey. & \\
\hline
\end{tabular}

In addition, mentors and mentees were queried about the frequency of meetings. Fifty percent of the mentor pairs met for the entire nine month period at least monthly; at times they met biweekly. One pair met once after several false starts and never met again. One group met from September 2014 to February 2015 only. At that time the mentee reported they had exhausted all of his questions.

All mentors and mentees then participated in a semi-structured interview at the end of the mentoring period. Field notes were generated during the interview and analyzed for common and contrasting themes.

\section{Mentee Perceptions}

All of the mentees noted their mentors were responsive; only one noted his mentor was only fairly responsive. This mentee noted "they both dropped the ball and only met once;" however, it was outlined at the beginning of the project that it was the mentee's responsibility to initiate contact with the mentor. One mentee noted his mentor initiated the contact consistently, All mentees talked with their mentors on the telephone at least once; $80 \%$ of the mentees noted they talked with their mentors on the telephone at least once per month and on some occasions more frequently. Only one mentee/mentor pair established formal goals; the remainder did not even though this was outlined in the mentor guide all participants received. It is interesting to note the group that established the goals were more satisfied overall with the mentor/mentee relationship.

All of the mentees noted the information they sought in this relationship was primarily information about their future careers. One mentee noted "it was helpful to talk to someone who had been in his shoes and was successful in his field." Eighty percent of the mentees noted they felt the mentor valued the relationship and they were compatible personally and professionally. The remaining mentee felt he and his mentor were compatible and had similar characteristics and interests; however, the mentor did not pursue the relationship with him so he did not know if the mentor valued the relationship or not. As noted previously, the responsibility for meetings fell to the mentees in this project. One half of the mentees noted they had gained additional skills from the relationship, while $80 \%$ noted the relationship was valuable and effective. One mentee noted he planned to keep in touch with his mentor and continue the mentoring relationship even outside of the formal program.

All of the mentees felt VineUp was overall an effective method for matching them with a mentor because it used personal and professional characteristics to provide the match. One mentee noted he liked that he could go into VineUp 
and view potential mentees and their biographies to select potential mentors. Two mentees noted they felt VineUp was very similar to LinkedIn.

\section{Mentors' Perceptions}

Overall, mentors perceived their mentees to be responsive. This varied from very responsive to "pretty responsive." All mentors noted they primarily discussed what it is like in an actual workplace and the skills that are needed to succeed above and beyond the formal engineering education experience like communication and teamwork. All reported they felt they were compatible personally and professionally with their mentees. One mentor did mentor two mentees in this project. Two of the three mentors noted they really felt valued by their mentees; one noted his mentee was "very sharp and probably did not need a mentor, but the relationship really unlocked ideas the mentee already had." The mentees that met with their mentors more frequently perceived the mentoring relationship to be more valuable.

Two of three of the mentors felt VineUp was not of any significant value, while the third thought it was very helpful to have everything in one place and go through a formalized matching process. One mentor felt a computer science major could probably create something similar that did not cost as much as the VineUp platform. One said he felt the mentoring program was not necessarily important to the company anymore; although they had actively recruited from this university in the past, due to budget cuts they had to cut over half of the universities they used to recruit from including the university where this study was conducted. This occurred during this pilot study. However, all mentors agreed it was better to do mentor/mentee matches based on a system that actually looks at personal and professional characteristics.

\section{Discussion}

The purpose of mentoring is to foster personal growth. In this pilot study all mentees reported some individual growth including the one that did not actively pursue the relationship. This is consistent with the findings of Levinson et al. in 1978. However, only one mentee felt he had actually gained any actual skills during the nine month mentoring period although Levinson et al noted skills can be realized by mentoring relationships. This could be attributed to the nature of the mentoring relationship: student and industry mentor. In addition the mentee/mentor pairs were geographically separate. Both of these factor could have contributed to the mentees overall view of this relationship being primarily an avenue where they could find out what it was like on the job rather than to gain actual skills. Instead, the mentees did learn what skills they would need that may not come from formal engineering education such as significant abilities to work in a team and communicate effectively. As noted by Straus et al. (2013), the more engaged the mentors and mentees where, the more valuable the relationship was perceived. The mentor characteristics present in this study as noted by Straus in addition to engagement were the professional experience level of the mentors, strategic advice, the emotional support and discussion of potential issues and pitfalls to prepare the mentees for the workplace. This project did "bridge the gap" between education and industry as noted by Khan and Gogos (2013).

Straus et al. (2013) also notes the mentee must be responsible, active listeners and must value the relationship. These characteristics were present in $80 \%$ of the mentees. One mentee did not drive the relationship; a critical function as noted by Straus. The right match was crucial in this pilot study as noted by Khan and Goges (2013) and the VineUp platform was perceived to be successful in creating compatible matches. The satisfaction level of the mentees was higher than the mentors as noted by Khan and Goges in regards to relationship perceptions and particular perceptions of the VineUp program.

The use of VineUp for this project did allow the mentoring process to transcend geographical barriers. As with most forms of e-mentoring, cost was incurred for the VineUp platform because it offered the matching process. Students perceived the matching process in VineUp to be of some value, while the mentors were mostly neutral. The majority of mentors noted the matching process could be done through other platforms or through a different matching process other than VineUp. Suggestions were made including Linked In, Skype, or a program-specific matching program created by the university internally.

\section{Conclusion}

The results of this study were primarily positive. Since this was a small pilot group, the university has decided to continue and expand the program in the 2015-2016 school year with a larger group of honor students and industry partners. The VineUp platform will be used and re-assessed. After this additional data is evaluated, the university will decide whether to continue with the VIneUp platform or initiate a different method to match mentors and mentees. This could include instruments such as the Myers Briggs, Grit Scores or other personality or professional habits assessment instruments. 


\section{References}

Beaty, C., Waters, Dziuvenis, G. \& Feldman, T. (2014). Use of professional industry interaction to enhance engineering education. Journal of Architectural Engineering, 20(3). http://dx.doi.org/10.1061/(ASCE)AE.1943-5568.0000148

Kapitzke, C. \& Hay, S. (2011). School education as social and economic governance: Responsibilizing communities through industry-school engagement. Educational Philosophy and Theory, 43 (10), 1103-1118. http://dx.doi.org/10.1111/j.1469-5812.2010.00679.x

Kasprisin, C.A., Single, P.B., Single, R.M. \& Muller, C.B. (2003). Building a better bridge: Testing e-training to improve e-mentoring programmes in higher education. Mentoring and Tutoring, 11(1), 67-78. http://dx.doi.org/10.1080/1361126032000054817

Khan, R., \& Gogos, A. (2013). Online mentoring for biotechnology graduate students: An industry-academia partnership. Journal of Asynchronous Learning Networks, 17(1), 89-107.

Kim, B., Jang, S., Jung, S., Lee, B., Puig, A. \& Lee, S. (2014). A moderated mediation model of planned happenstance skills, career engagement, career decision self-efficacy and career decision certainty. The Career Development Quarterly, 62, (1), 56-69. http://dx.doi.org/10.1002/j.2161-0045.2014.00070.x

Levinger, G. (1979). A social exchange view on the dissolution of pair relationships. In R. L. Burgess \& T. L. Huston (Eds.). Social exchange in developing relationships (pp. 169-196). New York: Academic Press. http://dx.doi.org/10.1016/B978-0-12-143550-9.50012-4

Lillian, T., Eby, J.E. \& Allen, T.D. (2011). Definition and evolution of mentoring. In T. T. Allen \& L.T. Eby’s The Blackwell handbook of Mentoring: A multiple perspectives approach. Malden, MA: Blackwell Publishing.

McCarthy, J. (2012). International design collaboration and mentoring for tertiary students through Facebook. Australasian Journal of Educational Technology, 28 (5), 755-775.

National Academy of Engineering. (2005). Educating the engineer of 2020: Adapting engineering education to the new century. Washington, D. C.: The National Academy Press.

Pinelli, T. E., Hall, C. W. \& Brush, K. M. (2013). Using assessments to determine the quality and effectiveness of a collaborative internship program in research. Quality Approaches in Higher Education, 4 (1), p. 5-12.

Straus, S.E., Johnson, M.O., Marquez, C. \& Feldman, M.D. (2013, January). Characteristics of successful and failed mentoring relationships: A qualitative study across two academic health centers. Academic Medicine, 88(1) 82-89. http://dx.doi.org/10.1097/ACM.0b013e31827647a0

VineUp. (2015). Retrieved from http://vineup.com/

Zey, M. (2011). Virtual mentoring. The challenges and opportunities of electronically-mediate formal mentoring programs. Review of Business Research, 11(4), 141-152. 